\title{
ARTICLE OPEN \\ Continuum understanding of twin formation near grain boundaries of FCC metals with low stacking fault energy
}

\author{
Jaimyun Jung ${ }^{1}$, Jae Ik Yoon ${ }^{1}$, Jung Gi Kim (D) ${ }^{1}$, Marat I. Latypov ${ }^{2}$ Jin You Kim ${ }^{3}$ and Hyoung Seop Kim (iD ${ }^{1,4}$
}

Deformation twinning from grain boundaries is often observed in face-centered cubic metals with low stacking fault energy. One of the possible factors that contribute to twinning origination from grain boundaries is the intergranular interactions during deformation. Nonetheless, the influence of mechanical interaction among grains on twin evolution has not been fully understood. In spite of extensive experimental and modeling efforts on correlating microstructural features with their twinning behavior, a clear relation among the large aggregate of grains is still lacking. In this work, we characterize the micromechanics of grain-to-grain interactions that contribute to twin evolution by investigating the mechanical twins near grain boundaries using a full-field crystal plasticity simulation of a twinning-induced plasticity steel deformed in uniaxial tension at room temperature. Microstructures are first observed through electron backscatter diffraction technique to obtain data to reconstruct a statistically equivalent microstructure through synthetic microstructure building. Grain-to-grain micromechanical response is analyzed to assess the collective twinning behavior of the microstructural volume element under tensile deformation. Examination of the simulated results reveal that grain interactions are capable of changing the local mechanical behavior near grain boundaries by transferring strain across grain boundary or localizing strain near grain boundary.

npj Computational Materials (2017)3:21; doi:10.1038/s41524-017-0023-1

\section{INTRODUCTION}

Twinning-induced plasticity (TWIP) steels in face-centered cubic metals contribute to the combination of high strength and ductility of high $\mathrm{Mn}$ steels with low stacking fault energy in the range of $20-50 \mathrm{~mJ} \mathrm{~m}^{-2}$. Although the mechanical twinning typically comprises only a small fraction of the material's total shear displacements, it acts as strong planar obstacles and induces a significant back-stress that increases the total flow stress through dislocation pile-ups. ${ }^{1}$ A number of studies are conducted in an effort to understand the formation and evolution of twin under the scope of grain size, ${ }^{2,3}$ chemical composition, ${ }^{2}$ grain orientation, ${ }^{4}$ and strain rate. ${ }^{5,6}$ Nevertheless, a thorough understanding of the mechanical nature of grain-to-grain interaction and its influence on twin formation has not been established yet. A growing body of evidence points to the significance of intergranular interactions on the formation of twins, particularly on the formation at grain boundaries ${ }^{4,7-9}$ due to the forced strain compatibility between adjacent grains activating multiple slip systems, which are proposed as prerequisites for mechanical twin formation. ${ }^{10,} 11$

Because grain interactions will be most evident near grain boundaries, a close evaluation of twin evolution near grain boundaries can provide a micromechanical picture of how grains interact to affect twin formation. Atomistic simulations are often performed to quantify specific dislocation-grain boundary reactions, ${ }^{12-14}$ which can be used to explain the nucleation of twins from boundaries. However, these quantifications are less effective when grain-to-grain interaction within a polycrystalline aggregate plays a significant role in mechanical twinning. In particular, when dealing with an arbitrary definition of grain boundary character distribution, a continuum approach is suitable to assess the role of forced strain compatibility among adjacent grains. While a continuum approach does not explicitly take into account all the necessary details associated with grain boundaries and dislocation structures, it is still an effective method to address the mechanical twins near grain boundaries due to the crystallographic and geometric relations among grains under any imposed deformation. In particular, prior studies on the microstructure-property linkage made with full-field calculations have proven to be successful in describing micromechanical behavior of polycrystalline materials. ${ }^{15-20}$

In this work, we take a continuum mechanics perspective to reveal the mechanical nature of grain interactions and the resulting twin activities near grain boundaries that are often observed with TWIP steels. To fully consider the grain interactions that are expected to play a major role in the formation of mechanical twins near grain boundaries, we have used a crystal plasticity simulation with microstructural features that are statistically equivalent to those observed under experimental measures. The synthetic microstructure of a hot-rolled TWIP (HRTWIP) steel is built with statistical data obtained from electron back-scattered diffraction (EBSD) technique. The simulation is carried out using a spectral-based full-field calculation of crystal plasticity constitutive model and associated boundary value problems with DAMASK. ${ }^{21}$ The results are then analyzed both macroscopically and microscopically to explore the general trend in mechanical twinning due to grain interactions. It is shown that grain interactions play a significant role in twinning behavior near grain boundaries of HRTWIP steels. In particular, the results

\footnotetext{
${ }^{1}$ Department of Materials Science and Engineering, Pohang University of Science and Technology (POSTECH), Pohang 37673, Republic of Korea; ${ }^{2}$ GT-CNRS UMI 2958, Georgia

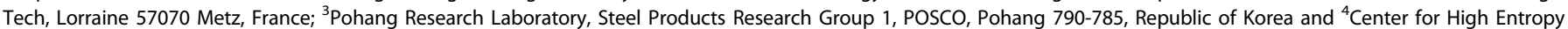
Alloys, Pohang University of Science and Technology (POSTECH), Pohang 37673, Republic of Korea Correspondence: Hyoung Seop Kim (hskim@postech.ac.kr)
}

Received: 28 September 2016 Revised: 24 April 2017 Accepted: 2 May 2017

Published online: 24 May 2017 
a

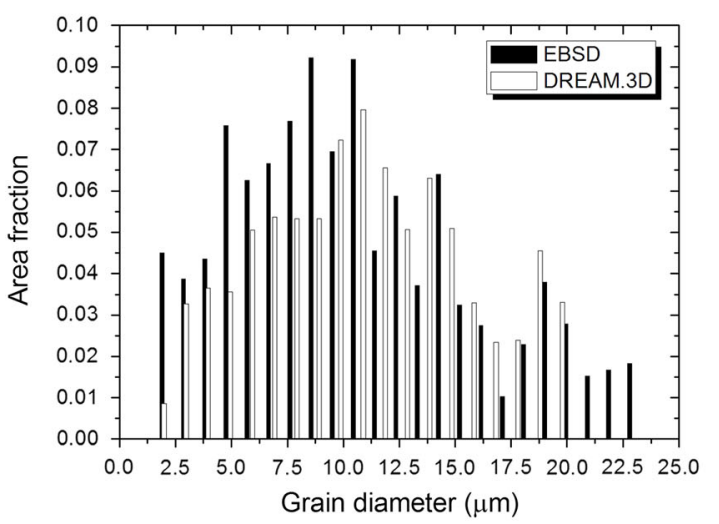

b



C

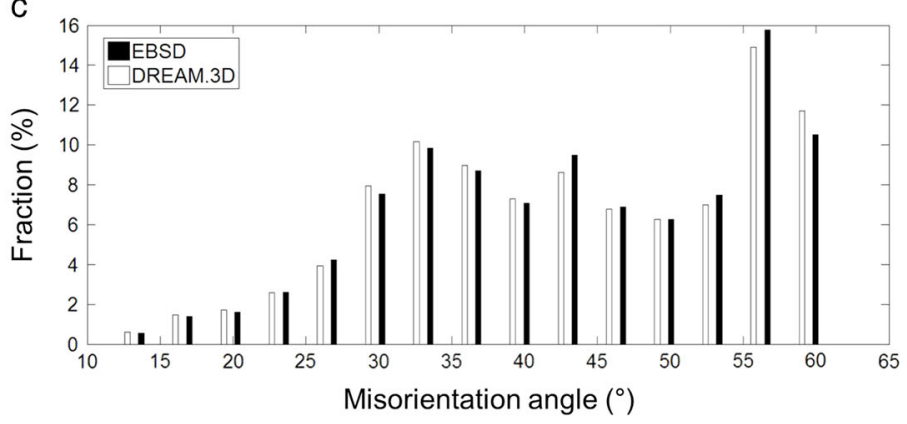

e


Fig. 1 Comparison between experimental (black) and synthetically generated (white) microstructural features: a grain size distribution; b grain aspect ratio; c misorientation distribution. The experimental and synthetically generated microstructures are visualized in $\mathbf{d}$ and $\mathbf{e}$, respectively

revealed how grains that are oriented in non-twin favoring orientations exhibit twinning behavior as a consequence of grain-grain interaction near their boundaries.

\section{RESULTS}

Statistical equivalence of the synthetic microstructure

The details of the reconstructed three-dimensional (3D) microstructure are illustrated in Fig. 1. The grain size distributions, major and minor axis lengths of each grain, and misorientation distributions of the experimentally observed and synthetically generated microstructures are shown in Figs. 1a-c, respectively. The average grain sizes of the experimentally observed and synthetically generated microstructures are comparable as shown in Table 1. In terms of grain shape, one can see a characteristic microstructure after rolling where the major axis (B) of each grain, which is tilted toward the rolling direction (RD), is longer than its minor axis (A) length, which is tilted toward the transverse direction. The difference in average aspect ratios of the grains
Table 1. Average grain sizes and aspect ratios measured from the experimentally observed and synthetic microstructures

\begin{tabular}{lcl}
\hline & $\begin{array}{l}\text { Average grain size } \\
\text { (standard deviation) }\end{array}$ & $\begin{array}{l}\text { Average aspect ratio } \\
\text { (standard deviation) }\end{array}$ \\
\hline $\begin{array}{l}\text { EBSD data } \\
\text { Synthetic } \\
\text { microstructure }\end{array}$ & $10.06(5.79) \mu \mathrm{m}$ & $0.489(0.096)$ \\
\end{tabular}

observed from EBSD image and synthetic microstructure are only 3\% (Table 1). The misorientation angle distributions of both the observed and synthetically generated microstructures are in good agreement and both exhibit characteristic peaks near $60^{\circ}$ due to annealing twin boundaries as shown in Fig. 1c. Figures1d, e show the EBSD images of the HRTWIP and synthetically generated HRTWIP, respectively. From Fig. 1 and Table 1, one can see that the microstructural features of the reconstructed and observed 
a

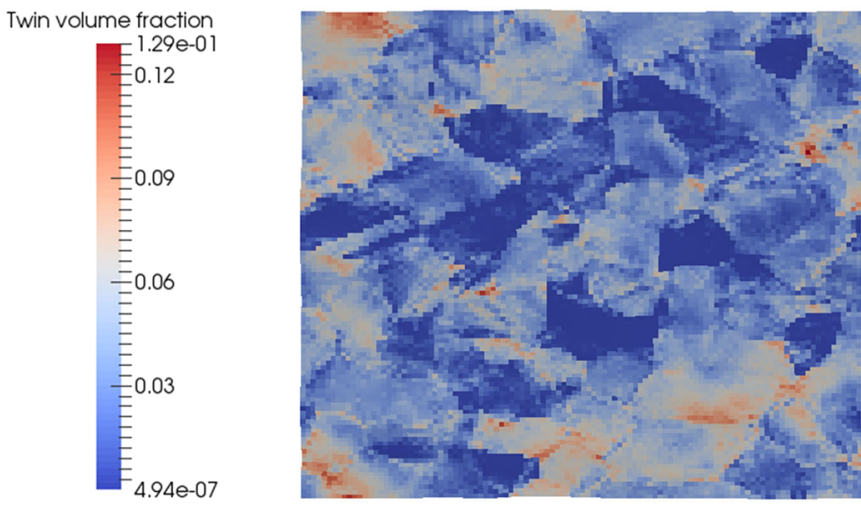

b
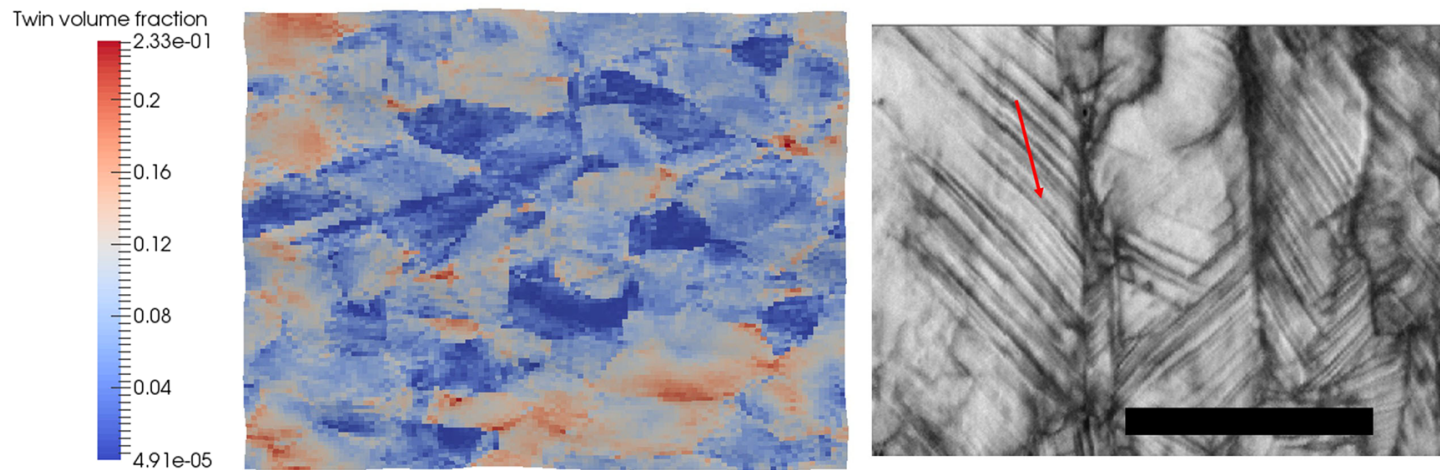

C
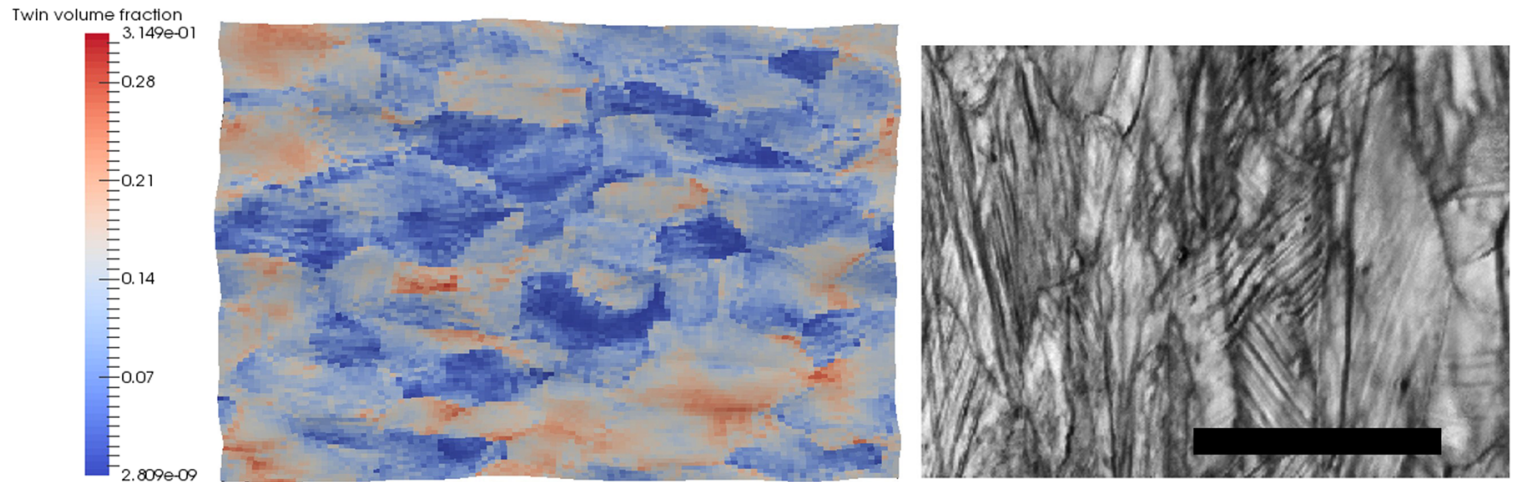

Fig. 2 Simulated (left) and experimental (right) results on twin volume fraction after tensile deformation. The experimental results are EBSD images based on $\mathrm{Cl}$ contrast. The scale bar is set to $10 \mu \mathrm{m}$ and the applied deformation was a $10 \%, \mathbf{b} 20 \%$, and c $30 \%$, respectively

microstructure are generally in good agreement. These features, which are sampled with 624 grains with $110 \times 110 \times 110$, or $1,331,000$, grid points, being statistically equivalent to the experimentally observed ones are sufficient to deliver realistic microstructures for HRTWIP steels. The details of the experimental characterization and simulation procedures are available in Materials and Methods.

\section{Twin activity near grain boundary}

From Figs. 2a-c, which show the simulated and experimental results on twin evolution after $10 \%, 20 \%$, and $30 \%$ elongations, respectively, one can observe that twin volume fraction (TVF) varies in both inter- and intra-granular manners. Some of the grains after 10 and $20 \%$ elongations exhibit strong twin activity while some grains exhibit limited twinned region. In particular, some twins take place locally near the grain boundary, which is indicated by a red arrow within the EBSD image (Fig. 2b). After
$30 \%$ elongation, most of the grains are twinned, but the twin activity varies from grain to grain. Investigation of twinning behavior near grain boundaries may provide significant insights as to how grain interactions affect the micromechanics of twinning because grain boundaries are where these interactions are the strongest. Of course, because the synthetic microstructure does not fully take into account the precise morphology and strengthening effect of sharp grain boundaries, the analysis made will not characterize the precise role of grain boundary energies and stress concentration. Instead, the twinning behavior of the first layer of elements constituting grain boundaries will be used to describe the role of grain interactions on twinning near the boundaries of each grain (Fig. 3).

In a macroscopic sense, twin seems to accumulate near grain boundaries first and then in grain interior at early stages of deformation. On average, TVF at grain boundaries is well above TVF within grain interior at earliest stages of deformation followed 
by similar TVF between grain interior and grain boundaries after intermediate stages of deformation (the ratios between near boundary TVF to grain interior TVF being 1.15, 1.08, 1.05, and 1.03 after $5 \%, 10 \%, 20 \%$, and $30 \%$ engineering strains, respectively). Given that small twinning is observed only after $5 \%$ elongation and that twin nucleation strain is around 3\% (ref. 7), the actual ratio between TVFs near boundary to interior will be around 1.15 at the onset of twin nucleation. Likely, this phenomenon may have stemmed from the fact that high stress concentration is mostly witnessed near grain boundaries. Figure 4 plots the frequency of resolved shear stress normalized by twin resistance $\left(\tau_{\text {resolved }} / \tau_{\text {twin }}\right)$ together with twin growth rate, which depends on the normalized shear stress after 5, 10, and $20 \%$ elongations. One can see that after $5 \%$ elongation, most of the normalized shear stress is well below 0.9 , which results in a negligible level of twin growth rate. Only $2.7 \%$ and $4.4 \%$ of the grain interior and boundary region

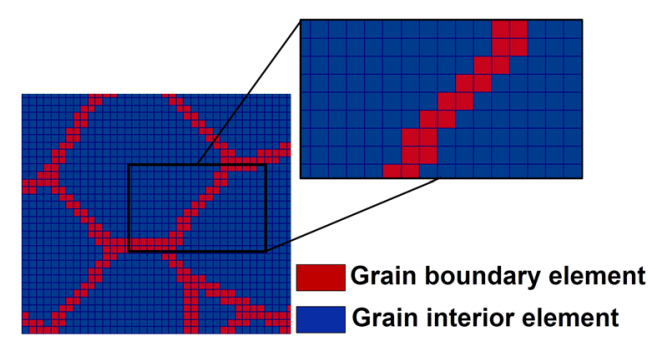

Fig. 3 Schematic illustration of grain interior and boundary elements. Red colored region represents grain boundary region while blue colored region represents grain interior region


exhibit shear stress above 0.9 , respectively. Nevertheless, these values still indicate that the twin activity near grain boundary is almost twice as high as that of grain interior. Meanwhile, after 10 and $20 \%$ elongations a large portion of shear stress is above 0.9 for both grain interior and grain boundary regions. In fact, after $10 \%$ elongation, $27.2 \%$ and $27.4 \%$ of the grain interior and grain boundary regions exhibit shear stress above $0.9 \%$, respectively. After $20 \%$ elongation, $32.4 \%$ and $32.8 \%$ of the grain interior and grain boundary experience shear stress above 0.9 , respectively. This correlates well with the results shown in Fig. 5a, which plots the average TVF of voxels normalized by its respective grain average TVF as a function of normalized distance to grain boundary after 5, 10, and 20\% elongations. Figure 5a shows that heterogeneous twin activity takes place at early stages of deformation, but the twin activity becomes more and more homogeneous upon further deformation. Therefore, the results in Figs. 4 and 5 a infer that stress localization near grain boundaries is responsible for the heterogeneity of twinning at early stages of deformation because most of the grain interior region experience a very low shear stress relative to twin resistance, but upon further deformation more fraction of grain interior region accumulates stress level high enough for notable twin activities and the normalized stress distribution becomes comparable with that of grain boundary region, resulting in a more homogeneous twin activity.

From Fig. 5b, one can see that individual grains can exhibit both larger and smaller TVFs at their boundaries than their interiors, demonstrating that grain interactions can either enhance or hamper twin activities on either side of the grain boundaries. If

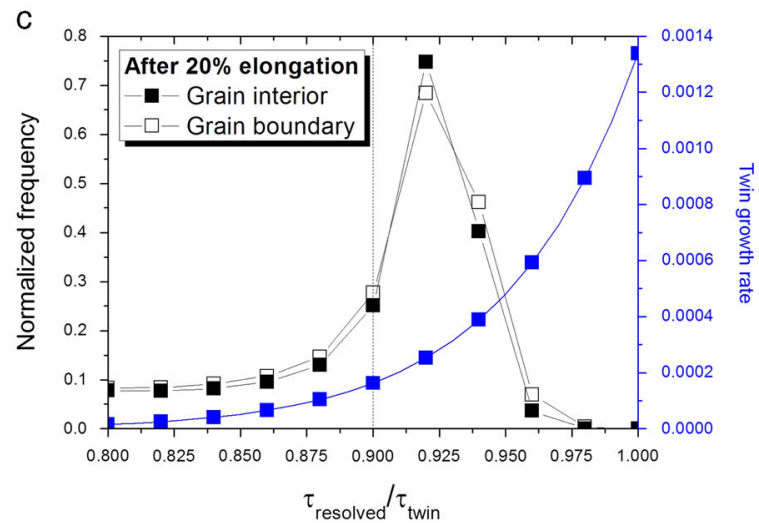

Fig. 4 Distribution of shear stress normalized by twin resistance of grain interior and grain boundary regions after a $5 \%$, b $10 \%$, and c $20 \%$ elongations, respectively. The curve colored in blue represents the twin growth rate against normalized shear stress value and the dotted line represents the point at which twin growth rate is more than $10 \%$ of the maximum growth rate 
a

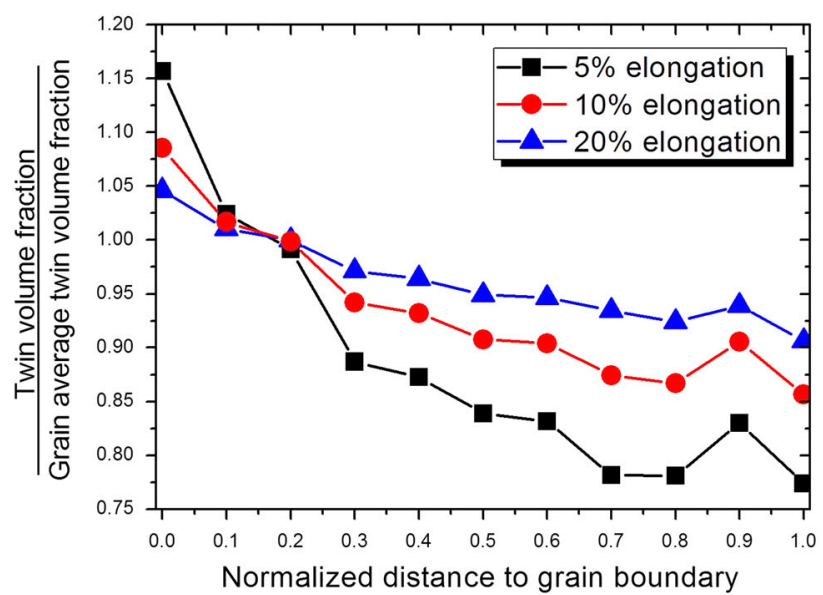

b

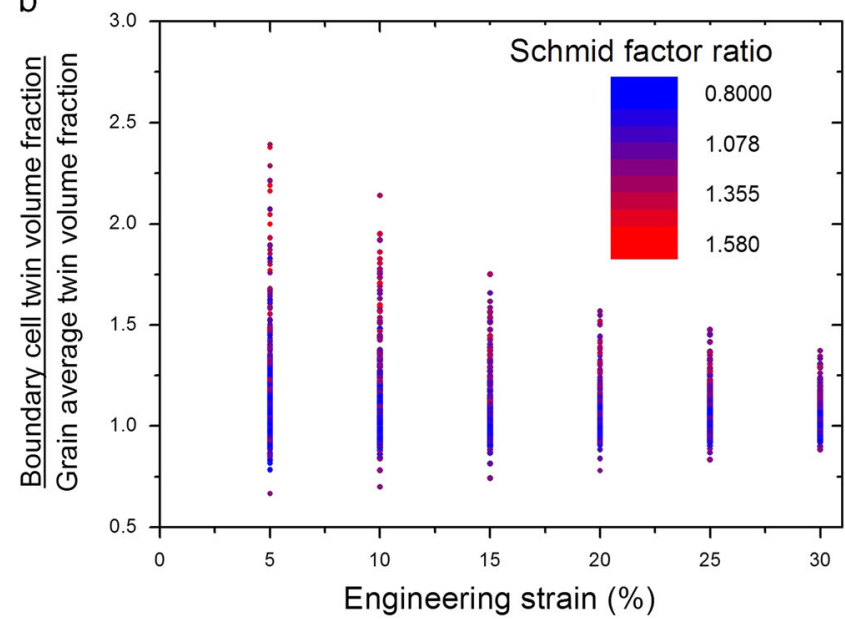

Fig. 5 Change in twinning behavior upon deformation: a Twin volume fraction normalized by grain average twin volume fraction plotted against normalized distance to grain boundary after $5 \%$ (black), $10 \%$ (red), and $20 \%$ (blue) elongations; b Twin volume fraction of grain boundary region normalized by its respective grain average twin volume fraction during tensile deformation up to $30 \%$ color coded by the Schmid factor ratio of each grain

individual grains are characterized by the ratio between Schmid factor of slip system and that of the twin systems (Schmid factor ratio), where higher Schmid factor ratio means that the stress state of the grains are resolved mainly on slip system instead of $<112>$ twin system, most of the grain boundaries that seem to hamper twinning belong grains with low Schmid factor ratio, or the twin favoring grains. In fact, for $76 \%$ of the grains with low TVFs near their boundaries have Schmid factor ratio less than 0.9 after $5 \%$ elongation. After $10 \%, 20 \%$, and $30 \%$ elongations $70 \%, 54 \%$, and $53 \%$ of the grains with boundaries that impede twin activity belong to grains with Schmid factor ratio less than 0.9 , respectively. On the other hand, majority of grains with medium to high Schmid factor ratio, or slip favoring grains, exhibit higher twin activities near their respective grain boundaries after $5 \%$ elongation. Further deformation up to 10,20 , and $30 \%$, large portion of grains with medium Schmid factor ratio no longer exhibit enhanced twin activities near their respective boundaries. These results indicate that while the initial orientation of each grain strongly affects the boundary properties in terms of twin activity, further deformation renders orientation of each grain alone insufficient to describe twin activity near grain boundaries, which is one of the main reasons why simple Schmid factor analysis results in notable deviations after later stages of deformation.

One key observation made is that a strong twin activity on one side of the boundary will likely result in strong twin activity near the boundaries of the other. Figure 6 illustrates this point by plotting average TVF near grain boundaries of each grain to that of the first layer of elements on the other side of the boundaries during the course of tensile elongation. The slope remains almost constant to 0.625 while the intercept changes to $0.0025,0.0102$, 0.028 , and 0.0439 after $5 \%, 10 \%, 20 \%$, and $30 \%$ elongations, respectively. This indicates that after $30 \%$ elongation, a grain with TVF above 0.1 near its grain boundary will exhibit lower twin activity near the boundary of the grain compared to that on the other side of the boundary. That is, the Schmid factor ratio is still strongly related to the slope of each line segment.

Given that TVF of one side of the boundary affects that of the other, one can come to the conclusion that the twin activity of a grain, in particular near the grain boundary of the grain, is strongly associated with its surrounding. Figure 7 plots the TVF near boundary normalized by grain average TVF and the ratio between Schmid factor ratio of the grain to that of its surrounding after $5 \%$, $10 \%, 20 \%$, and $30 \%$ elongations, respectively. One can see that

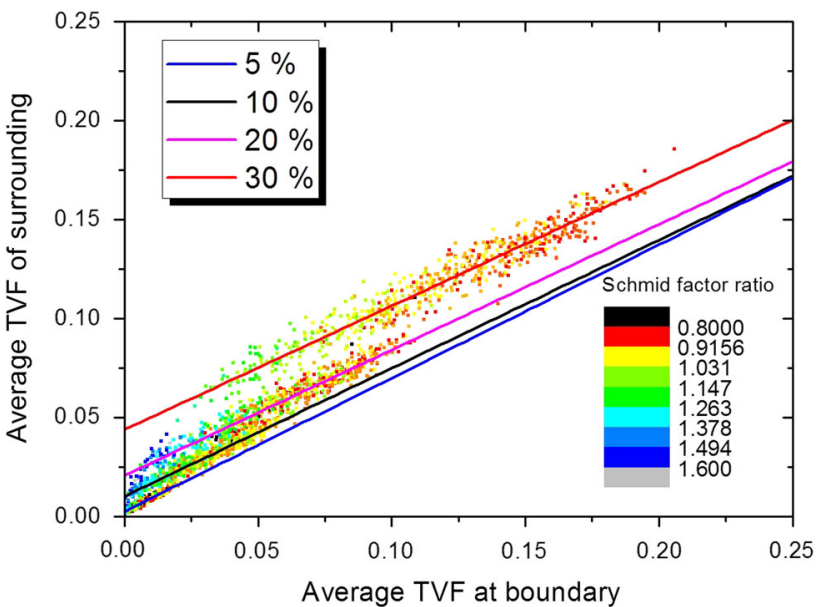

Fig. 6 Twin volume fraction of one side of the boundary to another during tensile deformation. The dotted data corresponds to that of individual grain color coded by its respective Schmid factor ratio. The straight lines colored in blue, black, magenta, and red represent averaged results after $5 \%, 10 \%, 20 \%$, and $30 \%$ elongations, respectively

when the surrounding of the grain is more twin favoring than the grain itself, then the grain will likely show larger twin activity near its boundary compared to its interior. Nevertheless, for grains surrounded by grains with higher Schmid factor ratio, the ratio between TVF near boundary to that within the interior region fluctuates near 1.0. Also, with increasing tensile elongation, the ratio between TVF near boundary to that within interior region of a grain decreases only for grains with twin favoring surrounding, which explains why macroscopically the ratio between TVFs near boundary to interior saturates close to 1.0.

Grain interactions that influence twin activity near grain boundary The results in Figs. 6 and 7 indicate that simply evaluating whether or not a grain is surrounded by grains with twin favoring orientations can provide a quick assessment of twin formation on grain boundaries. Nonetheless, the correlation made in Fig. 7 is relatively weak with large scatter of data. This is because while even under micromechanical stress state the Schmid factor ratio 
a

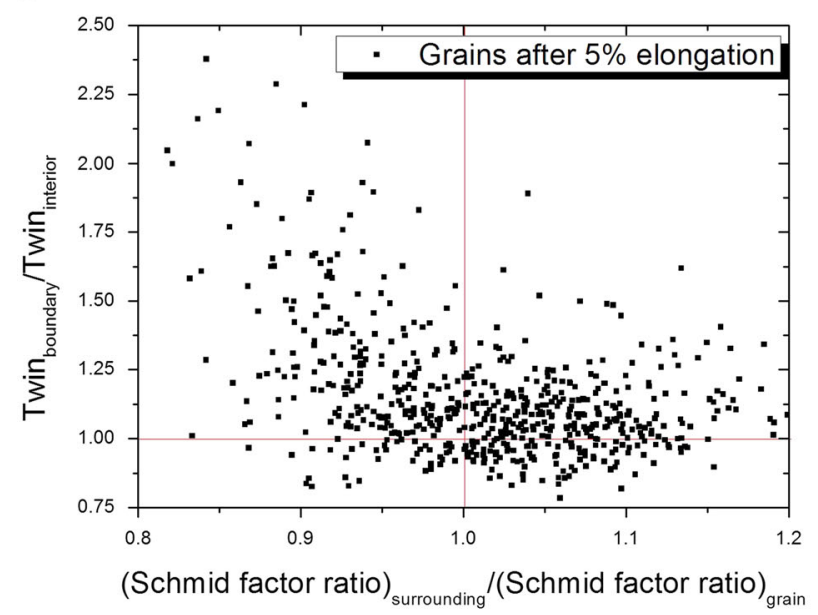

c



b

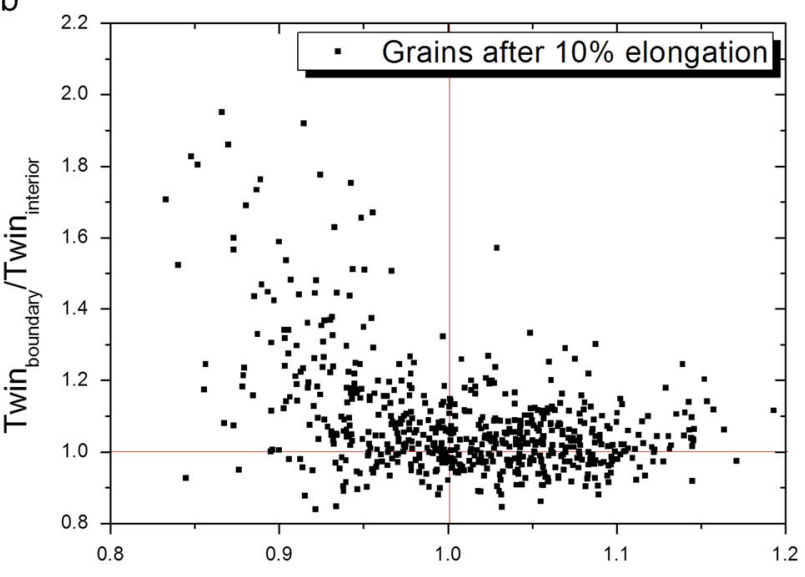

(Schmid factor ratio $)_{\text {surrounding }} /(\text { Schmid factor ratio })_{\text {grain }}$

d



Fig. 7 The ratio between twin volume fraction in grain boundary region to that within interior region of a grain plotted against the ratio between Schmid factor ratio of the grain to that of its surrounding after a $5 \%$, b $10 \%$, c $20 \%$, and d $30 \%$ elongations, respectively

of most grain interior regions did not deviate much from the Schmid factor ratio evaluated under macroscopic stress, near the vicinity of the boundaries of these grains, Schmid factor ratios can be significantly lowered, which would mean that the micromechanical stress states and orientations near the boundaries strongly deviate from macroscopic stress states and average orientations, respectively. As a consequence, higher twin activity is observed near the boundaries compared to that of the interior of the grains (Fig. 8).

The decreased Schmid factor ratio near the vicinity of grain boundaries of grains embedded within twin favoring surroundings (Fig. 8) indicates that the strain compatibility between grains is met by changing the local orientation and stress state near the grain boundaries. In particular, if the changes in local orientation and stress state increase the resolved stress on twin system, the twin formation near boundaries is expected. An extended interpretation of grain boundary kinematics proposed by Gurtin is utilized to quantify the driving force for enhanced or reduced twin activities near grain boundaries. ${ }^{22}$ The original construct of Gurtin's work employs the concept of Burgers tensor $(\boldsymbol{G})$ in the following form:

$$
\boldsymbol{G}=\left[\boldsymbol{H}_{\mathrm{B}}^{P}-\boldsymbol{H}_{\mathrm{A}}^{P}\right](\boldsymbol{n} \times),
$$

where $\boldsymbol{H}^{\boldsymbol{P}}$ denotes the plastic distortion caused by a slip-induced shear deformation within a grain, or Schmid tensor, while $\boldsymbol{n}$ represents grain boundary normal pointing grain $B$ from grain $A$. The magnitude of Burgers tensor serves as a measure of the accumulated defectiveness of the grain boundary due to the difference in plastic distortion. In fact, the free energy of the grain boundary will correlate with the squared value of the tensor. In essence, if the plastic distortion due to mechanical twins is incorporated, the tensor will then act as a driving force for twin formation on grain boundaries depending on how it is resolved on Schmid-orientation tensor $(\boldsymbol{N})$, defined below.

$\boldsymbol{N}_{\text {A or B }}^{a}=\boldsymbol{S}_{\text {A or B }}^{a}(\mathbf{n} \times)$,

Where $\boldsymbol{S}$ is the Schmid tensor of $a^{\text {th }}$ slip or twin system and $\boldsymbol{n}$ is the grain boundary normal. The resolved $\boldsymbol{G}$ on $\boldsymbol{N}$ in grain I ( $\mathrm{A}$ or B) is then represented as follows:

$\boldsymbol{N}_{l}^{\alpha}: \boldsymbol{G}=\sum_{\beta}\left[\left(\boldsymbol{N}_{l}^{\alpha}: \boldsymbol{N}_{\mathrm{B}}^{\beta}\right) \gamma_{\mathrm{B}}^{\beta}-\left(\boldsymbol{N}_{l}^{\alpha}: \boldsymbol{N}_{\mathrm{A}}^{\beta}\right) \gamma_{\mathrm{A}}^{\beta}\right]$

Where $\gamma_{\mathrm{B}}^{\beta}$ is the resolved shear strain on $\beta^{\text {th }}$ slip or twin system of grain B. While the original construct of Gurtin's formulation deals with dislocations, the main idea of Burgers tensor is extended in this work to incorporate twin induced plastic distortion. Gurtin, in Eq. (3), called the term $\boldsymbol{N}_{\mathrm{A}}^{\alpha}: \boldsymbol{N}_{\mathrm{B}}^{\beta}$ and $\boldsymbol{N}_{\mathrm{A}}^{a}: \boldsymbol{N}_{\mathrm{A}}^{\beta}$ the inter-grain interaction moduli $\left(C_{A B}^{\alpha \beta}\right)$ and intra-grain interaction moduli $\left(C_{A A}^{\alpha \beta}\right)$, 
respectively, as shown below.

$$
C_{l J}^{a \beta}=\left(m_{l}^{a} \cdot m_{J}^{\beta}\right)\left(n_{l}^{\alpha} \times \mathbf{n}\right) \cdot\left(n_{J}^{\beta} \times \mathbf{n}\right),
$$

where $m_{l}^{\alpha}, n_{l}^{\alpha}$, and $\boldsymbol{n}$ represent $a$ th slip/twin direction, slip/twin plane normal, and grain boundary normal of grain I, respectively. The inter-grain interaction moduli, which is essentially a geometrical transmission factor, is then maximally interactive if the absolute value is 1.0 and non-interactive if the value is 0 . Then, the relation given in Eq. (4) infers the following:

1. Plastic distortion of a grain that favors twin formation will enhance twin activities near grain boundaryof its neighbor if $C_{A B}^{a \beta}$ is high, which explains why certain grains, in particularn on-twin favoring grains that are surrounded by twin favoring grains, exhibit enhanced twin formation near their respective boundaries (Fig. 6).

2. The relative increase or decrease of Schmid factor ratio near grain boundaries, which is responsible for large scatter in Fig. 7, is due to the fact that plastic distortion of one side of the grain can either decrease or increase, respectively, the twin system on the other side.

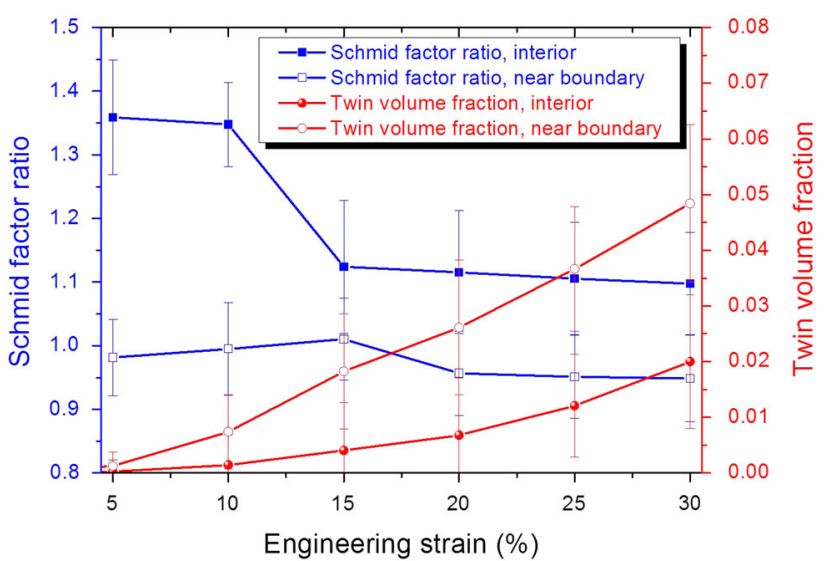

Fig. 8 Change in average Schmid factor ratio (blue) and twin volume fraction (red) of grain interior (solid symbol) and grain boundary (hollow symbol) regions with enhanced boundary twin activities. The error bars represent standard deviation of each averaged data
The sum of $C_{A B}^{\alpha \beta} \gamma_{B}^{\beta}$ of each grain against the difference between near grain boundary TVF and grain interior TVF after 5\% elongation is plotted to characterize the grain interactions that influence twinning near grain boundaries (Fig. 9). We have limited our attention to (1) grains with very high and very low TVFs to better distinguish how large heterogeneity leads to different twinning behavior near grain boundaries, (2) grain boundaries that are larger than half the average grain size because small grain boundaries may be affected by grain interactions present in other grain boundaries, and (3) grain boundaries with constant boundary normal vectors for simplification.

Two distinguished types of grain interactions are found. First type of interaction mainly occurred with grain pairs that exhibit large difference in twin activities. In this type of interaction, increasing inter-grain interaction moduli increases the near grain boundary TVFs of less twin favoring grains (Fig. 9a). This is because the twin favoring grains are typically "hard" grains (high Taylor factor) compared with grains that exhibit very low twin activities so a hard boundary condition is imposed on the Taylor "soft" grains. When the hard grains activate slip/twin systems with high interaction moduli, slip and twin-induced plastic distortions are effectively resolved on twin systems near the grain boundaries of the less twin favoring grains, which would activate twin systems.

The second type of grain interactions is much more frequently observed compared to the first. This type of interaction is observed when the TVFs of adjoining grains are not significantly different. In this case, twin activities near grain boundaries are largely enhanced when the interaction moduli are very low between two grains with low twin activities. When limited strain transfer, either by slip or twin, occurs across a grain boundary, strain localization takes place on both grains near the grain boundary. While the localization does not guarantee activation of twin systems, illustrated by large scatter of data in Fig. 9 with decreasing twin activity near grain boundary, it still may cause localized rotation or stress accumulation that can activate twin systems. It is worth noting that localization can also lead to reduced twin activity near a grain boundary, but this mostly applies to adjoining grain pairs with high TVFs (above twice the polycrystalline average). Thus, the decrease is very low relative to the TVFs of the grains on either side of the grain boundary.

\section{DISCUSSION}

When two grains with one grain with highly twin-favoring orientation are separated by low grain boundary misorientation,
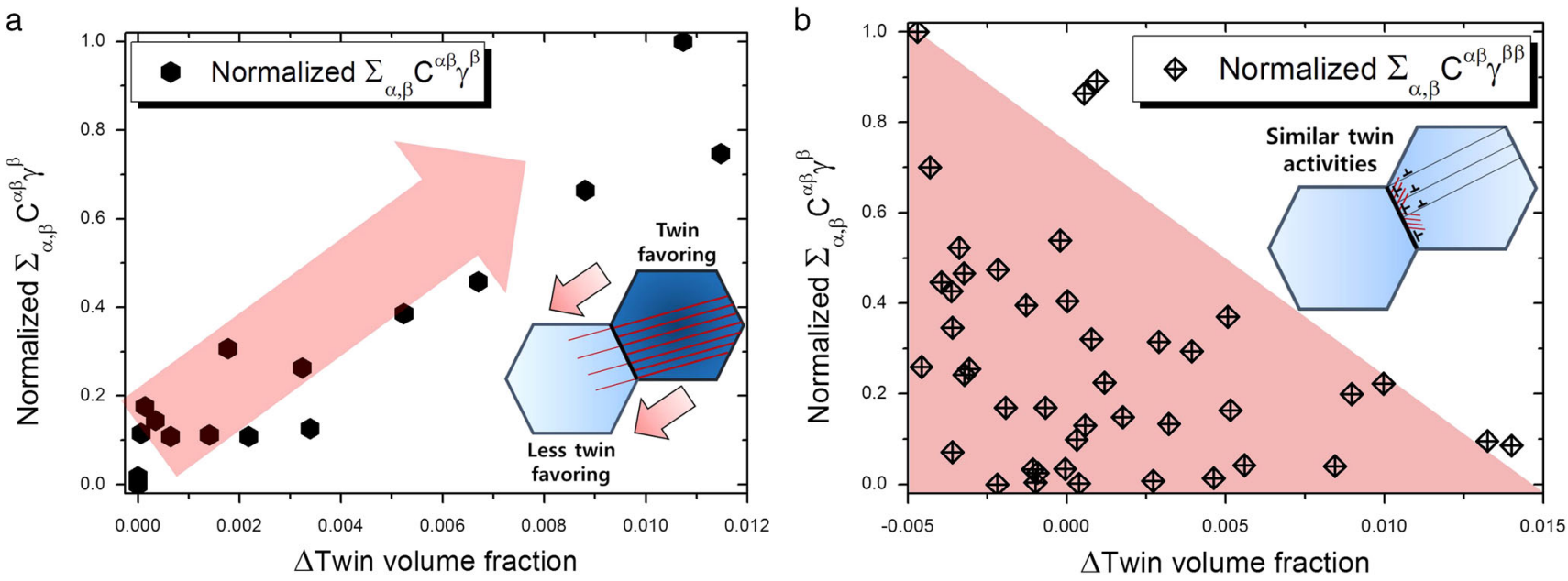

Fig. 9 Calculated inter-grain interaction coefficients against the difference between twin volume fraction of grain boundary and grain interior regions due to $\mathbf{a}$ strain transfer and $\mathbf{b}$ strain localization. The inset illustrates how $\mathbf{a}$ strain transfer and $\mathbf{b}$ strain localization affect twinning near grain boundaries 
twin propagation from one grain to another is possible. ${ }^{23,24}$ This, of course, is not sufficient to explain the trend illustrated in Figs. 7 and 8 . Gutierrez-Urrutia et al. ${ }^{7}$ reported that some grains that are adjacent to highly twinned grains and oriented close to $<100>/ /$ tensile axis directions exhibited twinning behavior near their respective boundaries that stand between them and highly twinned grains. They have argued that grains that are unfavorably oriented for mechanical twins exhibit twinning behavior due to (i) local shear stress concentration of twinned neighboring grain and (ii) difference in local stress state compared to the macroscopic one after high tensile strain. The results seen in Fig. 7 further support that high twin activity near the grain boundary strongly depends on the local deformation state and crystallographic orientation of the adjacent grains.

To demonstrate how grain boundary kinematics can influence near boundary twin formation, we have employed inter-grain interaction moduli, which is a geometrical slip/twin transmission factor, and proposed two types of grain interactions, one of which enhances twin formation near grain boundaries via transfer of strain and the other that enhances twin formation near grain boundaries via localization (Fig. 9).

We establish that, in terms of mechanical deformation, the involvement of twins on one side of the boundary will contribute to the twin formation on the other side only when there exists a large difference twin activities between the grains on either side of the boundary and geometric transmission factor is high enough, meaning that misorientation and boundary topology will play significant roles in determining the deformation systems that interact to affect twin formation. In most cases, however, near grain boundary twinning seems to be correlated with strain localization due to limited slip/twin transfer among grains. While the interpretation of twin formation on grain boundaries does not incorporate full details of dislocation structures and grain boundary strengthening, we emphasize that the kinematics of grain boundaries provides a mechanical driving force near the grain boundaries that need to be taken into account in future studies on grain interaction and its influence on deformation twinning. The present study provides a basis for grain interactions that contribute to twin formation near grain boundaries in a continuum framework and opens up new opportunities in designing microstructure via manipulating the mechanical interactions among microstructural features to maximize twin formation.

\section{MATERIALS AND METHODS}

The TWIP steel sheets with a thickness of $2 \mathrm{~mm}$ were acquired from POSCO. The composition of the steel is $0.6 \mathrm{C}-15 \mathrm{Mn}-1.2 \mathrm{Al}$ (wt\%). The tensile test was conducted to obtain the stress-strain curve of the specimen using a servo-hydraulic universal testing machine (UTM, model 1361, Instron Co., USA) at room temperature. The tensile test in the rolling direction (RD) was made with dog bone-shaped plate specimens with a 5.0, 2.5, and $1.0 \mathrm{~mm}$ in gage length, gauge width, and thickness, respectively, at a strain rate of $0.001 \mathrm{~s}^{-1}$. During all tensile tests, the strains were measured with the digital image correlation (DIC) method in an optical strain gauge system (ARAMIS $5 \mathrm{M}, \mathrm{GOM} \mathrm{mbH}$, Germany). The DIC technique is known to offer highly accurate and precise strain measures. ${ }^{25,} 26$ After the tensile test for the stress-strain curve, four tensile specimens were analyzed after 5, 10, 20, and $30 \%$ of engineering strains to measure the microstructure and microtexture evolutions with EBSD technique.

Samples for EBSD were prepared by mechanical polishing up to 1200 grit in US mesh followed by electrolytic polishing with $8 \%$ perchloric acid solution. Field emission gun XL30S scanning electron microscope at $25 \mathrm{keV}$ was used for the EBSD measurements. Additional EBSD measurements on 10,20 , and $30 \%$ elongated samples were conducted with higher resolution with a step size of $50 \mathrm{~nm}$ to resolve twins that have grown.

The EBSD orientation maps provided estimates of twin evolution after the tensile elongation. The twin boundaries are defined as $\left.\sum 3=60^{\circ}<111\right\rangle$. The maximum misorientation angle was identified by $\Delta \theta \leq 15^{\circ} \Sigma^{-\frac{5}{6}}$ with a tolerance angle of $6^{\circ}$. EBSD measurements on the evolution of TVF during tension was based on point counting analysis. ${ }^{27}$ The evolution of twin fraction from simulated results fall within the range of published results. ${ }^{4,}{ }^{28}$ Also, while the EBSD measurements are not capable of resolving single mechanical twin, they are capable of resolving grown twin or a number of twins in a bundle. Thus, the EBSD measurements are still capable of providing a qualitative picture on the evolution of twin fraction.

The EBSD images built a statistical dataset using DREAM.3D that was used to construct a synthetic 3D microstructure with statistically equivalent features including grain size distribution, misorientation distribution, grain shape, orientation distribution, and annealing twins. ${ }^{29}, 30$ The virtual 3D microstructure was then simulated under tensile load with crystal plasticity model proposed by Kalidindi. ${ }^{31}$ The simulation was conducted with DAMASK. ${ }^{21}$

The twin-incorporated crystal plasticity model follows the concepts outlined by Kalidindi. ${ }^{31}$ In Kalidindi's work, twinning is incorporated as an additional kinematic degree of freedom for shear by decomposing the plastic velocity gradient in the following manner:

$L_{p}=\left(1-\sum_{\beta}^{N^{\mathrm{tw}}} f^{\beta}\right) \sum_{a}^{N^{s}} \dot{\gamma}^{\alpha} S_{s l}^{\alpha}+\sum_{\beta}^{N^{\mathrm{w} w}} \dot{f}^{\beta} \gamma^{\mathrm{tw}} S_{\mathrm{tw}}^{\beta}$

$S=m \otimes n$,

where, $\dot{\gamma}^{\alpha}, \gamma^{\text {tw }}, f^{\beta}$, and $\dot{f}^{\beta}$ denote plastic shearing rate, constant twin shear, twin fraction at twin system $\beta$, and twinning rate, respectively. $N^{s}$ and $N^{\text {tw }}$ refer to the total number of slip and twinning system, respectively, and $S^{s}$ and $S^{\text {tw }}$ denote slip and twinning systems, respectively.

Also, slip-twin and twin-twin interactions are understood via phenomenological hardening rates while TVF evolves through the following equation, which is much similar to how slip-induced shear evolves, which is shown in Eq. (7) below, in power law form:

$\dot{\gamma}^{a}=\dot{\gamma}_{0}\left|\tau^{a} / s^{a}\right|^{1 / m} \operatorname{sign}\left(\tau^{a} / s^{a}\right)$,

$\dot{f}^{\beta}=\frac{\dot{\gamma}_{0}}{\gamma^{\mathrm{tw}}}\left(\tau^{\beta} / s_{\mathrm{tw}}^{\beta}\right)^{1 / m}$, where $f^{\beta}>0$ if $\tau^{\beta}>0$,

where, $\dot{\gamma}_{0}, \tau^{\beta}$, and $s_{\mathrm{tw}}^{\beta}$ refer to reference shear rate, resolved shear stress, and twin resistance on $\beta$ twin system, respectively. The hardening law associated with slip and twin systems is described as follows:

$\dot{s}^{a}=h_{s}\left(1-s^{a} / s_{s}^{\varepsilon}\right) \sum_{s l i p} \dot{\gamma}^{k}$, where $s_{s}^{a}=s_{s 0}+s_{\mathrm{pr}}\left(\sum_{k} f^{k}\right)^{0.5}$,

$\dot{s}^{\beta}=h_{0}^{\mathrm{tw}}\left(\sum_{a} f^{a}\right)^{b} \sum_{k} \gamma^{\mathrm{tw}} \dot{f}^{k}$,

where $\dot{s}^{a}, \dot{s}^{\beta}, s_{s 0}, h_{s}, h_{0}^{t w}$, and $s_{\mathrm{pr}}$ refer to change in slip resistance, twin resistance, saturation value associated with slip system, hardening rate of slip and twin systems, and hardening parameters associated with phenomenological interactions between slip and deformation twinning, respectively. Once the TVF evolves, its effect on slip resistance and twin resistance is defined by the hardening law in Eqs. (9) and (10), and the change in elasticity tensor takes place as follows:

$C_{i j k l}^{\text {twinned }}=C_{p q r s}^{\text {untwinned }} Q_{i p} Q_{j q} Q_{k r} Q_{l s}$,

Where $C_{i j k l}^{t w i n n e d}$ and $C_{\text {pqrs }}^{\text {untwinned }}$ are the elasticity tensors of the twinned and untwinned regions, respectively, and $Q$ is the transformation matrix between the lattice orientation in the untwinned region to that in the twinned regions.

The Cauchy stress $(\sigma)$ in the crystal is assumed to be volume average of stresses in untwinned and twinned regions as

$\sigma=\left(1.0-\sum_{\beta} f^{\beta}\right) \sigma^{\mathrm{mt}}+\left(\sum_{\beta} f^{\beta}\right) \sigma^{\mathrm{tw}}$.

The parameters used in the simulations are $170,240,5000,750,750$, and $400 \mathrm{MPa}$ for $s_{0, \text { slip, }} s_{0, \text { twin }} h_{0}^{\mathrm{tw}}, h_{\mathrm{s},} s_{\mathrm{s} 0 \text {, }}$ and $s_{\mathrm{pr}}$ respectively. These parameters are fitted through repetitive simulations to match the average von Mises stress-tensile strain to experimental true stress-strain curves. The elastic constants for the HRTWIP are 175,115 , and $135 \mathrm{GPa}$ for $C_{11}, C_{12}$, and $C_{44}$, respectively. Macroscopic stress-strain responses for 727 grains with $115 \times$ $115 \times 115$ grid points and 951 grains with $125 \times 125 \times 125$ grid points were also simulated to ensure that the average mechanical response had converged for 624 grains. The experiment and simulation results on macroscopic properties are provided in the supplementary material. It is worth mentioning that the model used does not explicitly take into account the precise morphology and arrangement of mechanical twins but rather the volume fraction of twins at each material point. Nonetheless, 
because the focus of this study is on how mechanical interactions among grains affect the general trend in twin activities near grain boundaries, we believe that the model used is suitable for the present study.

\section{ACKNOWLEDGEMENTS}

This study was supported by POSCO (2015Y073), Brain Korea 21 PLUS project for Center for Creative Industrial Materials (F16SN25D1706), and National Research Foundation of Korea (NRF) grant funded by the Korean government (MISP) (No. 2014R1A2A1A10051322).

\section{AUTHOR CONTRIBUTIONS}

The project was planned and supervised by H.S.K. and J.J. Data collection and analysis were performed by J.I.Y., M.I.L., and J.J. Samples were designed and prepared by J.G.K. and J.Y.K. The manuscript was prepared by H.S.K. and J.J.

\section{COMPETING INTERESTS}

The authors declare that they have no competing financial interests.

\section{REFERENCES}

1. Bouaziz, O., Allain, S., Scott, C. P., Cugy, P. \& Barbier, D. High manganese austenitic twinning induced plasticity steels: A review of the microstructure properties relationships. Curr. Opin. Solid St. M 15, 141-168 (2011).

2. Pierce, D. T., Jimenez, J. A., Bentley, J., Raabe, D. \& Wittig, J. E. The influence of stacking fault energy on the microstructural and strain hardening evolution of Fe-Mn-Al-Si steels during tensile deformation. Acta Mater. 100, 178-190 (2015).

3. Kang, S., Jung, J. G., Kang, M., Woo, W. \& Lee, Y. K. The effects of grain size on yielding, strain hardening, and mechanical twinning in Fe-18Mn-0.6C-1.5Al twinning-induced plasticity steel. Mater. Sci. Eng. A 652, 212-220 (2016).

4. Beladi, $\mathrm{H}$. et al. Orientation dependence of twinning and strain hardening behaviour of a high manganese twinning induced plasticity steel with polycrystalline structure. Acta Mater. 59, 7787-7799 (2011).

5. Sun, C. Y., Guo, N., Fu, M. W. \& Wang, S. W. Modeling of slip, twinning and transformation induced plastic deformation for TWIP steel based on crystal plasticity. Int. J. Plasticity 76, 186-212 (2016).

6. Song, B.-H., Kim, J., Jeong, S., Choi, I. \& Lee, Y.-K. Effect of deformation twin on mechanical properties of a lean manganese twinning induced plasticity (TWIP) steel deformed at quasi-static strain rates. Korean J. Met. Mater. 52, 11-19 (2014).

7. Gutiérrez-Urrutia, I., Zaefferer, S. \& Raabe, D. The effect of grain size and grain orientation on deformation twinning in a Fe-22 wt\% Mn-0.6wt.\% C TWIP steel. Mater. Sci. Eng. A 527, 3552-3560 (2010).

8. Beladi, H., Nuhfer, N. T. \& Rohrer, G. S. The five-parameter grain boundary character and energy distributions of a fully austenitic high-manganese steel using three dimensional data. Acta Mater. 70, 281-289 (2014).

9. Ullrich, C. et al. Interplay of microstructure defects in austenitic steel with medium stacking fault energy. Mater. Sci. Eng. A 649, 390-399 (2016).

10. Karaman, I., Sehitoglu, H., Gall, K., Chumlyakov, Y. I. \& Maier, H. J. Deformation of single crystal Hadfield steel by twinning and slip. Acta Mater. 48, 1345-1359 (2000).

11. Mahajan, S. \& Chin, G. Y. Formation of deformation twins in f.c.c. crystals. Acta Metall 21, 1353-1363 (1973).

12. $\mathrm{Yu}, \mathrm{W}$. \& Shen, S. Energetic of point defect interacting with grain boundaries undergone plastic deformations. Int. J. Plasticity 85, 93-109 (2016).

13. Xu, S., Xiong, L., Chen, Y. \& McDowell, D. L. Sequential slip transfer of mixedcharacter dislocations across $\Sigma 3$ coherent twin boundary in FCC metals: a concurrent atomistic-continuum study. Npj Comput. Mater 2, 15016 (2016).

14. Zhang, L., Lu, C. \& Tieu, K. Atomistic simulation of tensile deformation behavior of $\Sigma 5$ tilt grain boundaries in copper bicrystal. Sci. Rep. 4, 5919 (2014).
15. Tari, V. et al. The effect of deformation twinning on stress localization in a three dimensional TWIP steel microstructure. Modelling Simul. Mater. Sci. Eng 23 045010 (2015).

16. Zhang, H., Diehl, M., Roters, F. \& Raabe, D. A virtual laboratory using high resolution crystal plasticity simulations to determine the initial yield surface for sheet metal forming operations. Int. J. Plasticity 80, 111-138 (2016).

17 Lee, D. J. et al. Three-dimensional real structure-based finite element analysis of mechanical behavior for porous titanium manufactured by a space holder method. Comp. Mater. Sci 100, 2-7 (2015).

18. Jung, J. M., Yoo, J. H., Jeong, H. J., Lee, S. \& Kim, H. S. Three-dimensional characterization of $\mathrm{SiC}$ particle-reinforced Al composites using serial sectioning tomography and thermos-mechanical finite element simulation. Metall. Mater. Trans. A 45, 5679-5690 (2014).

19. Yeratapally, S. R., Glavicic, M. G., Hardy, M. \& Sangid, M. D. Microstructure based fatigue life prediction framework for polycrystalline nickel-base superalloys with emphasis on the role played by twin boundaries in crack initiation. Acta Mater. 107, 152-167 (2016).

20. Farukh, F. et al. Realistic microstructure-based modelling of cyclic deformation and crack growth using crystal plasticity. Comp. Mater. Sci 111, 395-405 (2016).

21. Roters, F. et al. DAMASK: the Düsseldorf advanced material simulation kit for studying crystal plasticity using an FE based or a spectral numerical solver. Procedia IUTAM 3, 3-10 (2012).

22. Gurtin, M. E. A theory of grain boundaries that accounts automatically for grain misorientation and grain-boundary orientation. J. Mech. Phys. Solids 56, 640-662 (2008).

23. Fernández, A., Jérusalem, A., Gutiérrez-Urrutia, I. \& Pérez-Prado, M. T. Threedimensional investigation of grain boundary-twin interactions in a Mg AZ31 alloy by electron backscatter diffraction and continuum modeling. Acta Mater. 61, 7679-7692 (2013).

24. Kumar, M. A., Kanjarla, A. K., Niezgoda, S. R., Lebensohn, R. A. \& Tomé, C. N. Numerical study of the stress state of a deformation twin in magnesium. Acta Mater. 84, 349-358 (2015).

25. Yoon, J. I. et al. Obtaining reliable true plastic stress-strain curves in a wide range of strains using digital image correlation in tensile testing. Korean J. Met. Mater. 54, 231-236 (2016)

26. Shin, J.-H., Jahanzeb, N., Kim, M.-S., Hwang, J.-H. \& Choi, S.-H. Analysis of deformation and failure behaviors of TIG welded dissimilar metal joints using miniature tensile specimens. Korean J. Met. Mater. 55, 125-131 (2017).

27. Renard, K. \& Jacques, P. J. On the relationship between work hardening and twinning rate in TWIP steels. Mater. Sci. Eng. A 542, 8-14 (2012).

28. Saleh, A. A. \& Gazeder, A. A. A re-evaluation of "The micromechanics of twinning in a TWIP steel". Mater. Sci. Eng. A 649, 184-189 (2016).

29. Groeber, M. A. \& Jackson, M. A. DREAM.3D: a digital representation environment for the analysis of microstructure in 3D. Integr. Mater. Manuf. Innov 3, 5-22 (2014).

30. Kim, S.-H. \& Rhyim, Y. Serial sectioning and reconstruction techniques for threedimensional microscopy of metallic materials. Korean J. Met. Mater. 54, 698-710 (2016).

31. Kalidindi, S. R. Incorporation of deformation twinning in crystal plasticity models. J. Mech. Phys. Solids 46, 267-290 (1998).

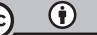

Open Access This article is licensed under a Creative Commons Attribution 4.0 International License, which permits use, sharing, adaptation, distribution and reproduction in any medium or format, as long as you give appropriate credit to the original author(s) and the source, provide a link to the Creative Commons license, and indicate if changes were made. The images or other third party material in this article are included in the article's Creative Commons license, unless indicated otherwise in a credit line to the material. If material is not included in the article's Creative Commons license and your intended use is not permitted by statutory regulation or exceeds the permitted use, you will need to obtain permission directly from the copyright holder. To view a copy of this license, visit http://creativecommons. org/licenses/by/4.0/.

(c) The Author(s) 2017

Supplementary Information accompanies the paper on the npj Computational Materials website (doi:10.1038/s41524-017-0023-1). 